\title{
Accretion in the broad line region of active galactic nuclei
}

\author{
E. Bon, ${ }^{1}$ L. Č. Popović ${ }^{1}$ and D. Ilić ${ }^{2}$ \\ ${ }^{1}$ Astronomical Observatory, 11160 Belgrade, Serbia \\ ${ }^{2}$ Department of Astronomy, University of Belgrade, 11000 Belgrade, Serbia \\ email: ebon@aob.bg.ac.yu, lpopovic@aob.bg.ac.yu,dilic@matf.bg.ac.yu
}

\begin{abstract}
We modeled the single-peaked Broad Emission Lines (BELs) with two-component model (accretion disk, with surrounding spherical region), comparing it with observational line profiles for a number of Active Galactic Nuclei (AGN). We find that the accretion in the Broad Line Region (BLR) can be present even if the profiles of BELs are single-peaked.
\end{abstract}

Keywords. Galaxies: nuclei - galaxies: Seyfert - accretion, accretion disks - quasars: emission lines

\section{Introduction}

The BLR is a very attractive subject for studding since the most widely accepted model for AGN includes a super-massive black hole and an accretion disk. Some AGN emit double-peaked lines, indicating the existence of the disk emission, but their number is small and statistically insignificant (around 5\%, see Eracleous \& Halpern 2003). Majority of AGN with one-peaked BELs, but it does not necessary indicate that the contribution of the disk emission can be neglected. Even if the emission in a spectral line comes only from a disk, the parameters of the disk (e.g. for a small inclination angle) can produce a single-peaked line (Chen \& Halpern 1989, Dumont \& Collin-Souffrin 1990, Kollatschny \& Bischoff 2002, Kollatschny 2003).

Recently (Popović et al. 2001, Popović et al. 2002, Popović et al. 2003, Popović et al. 2004, Ilić, D. et al. 2006, Bon et al. 2006) showed that broad emission lines of several AGN, can be well fitted with a model which has two components.

In this work we study the possibility to have the disk emission in the BLR of an AGN with the single-peaked BELs.

\section{Two-component model for the BLR}

The two component model consists of an accretion disk (Keplerian relativistic model Chen \& Halpern 1989), and emission of surrounding spherical region. Two component model is described in Popović et al. 2004. Here we did a simulation of the line profiles with different parameters of the model.

We simulated and measured parameter $k=W_{1 / 10} / W_{1 / 2}\left(W_{1 / 2}=\right.$ full width at a half maximum, $W_{1 / 10}=$ full width at a tenth of maximum) for different flux contributions of the disk and spherical region. We concluded that the parameter $k$ can be very useful as an indicator of the disk emission. Even if we have a BEL with more prominent single-peak, there is still a possibility that the disk emission is present. Moreover, we find that the disk emission can be detected if the ratio of the disk emission flux $\left(F_{\text {sph }}\right)$ and the flux of the spherical region $\left(F_{\text {disk }}\right)$ is $F_{\text {disk }} / F_{\text {sph }}>0.5$. 


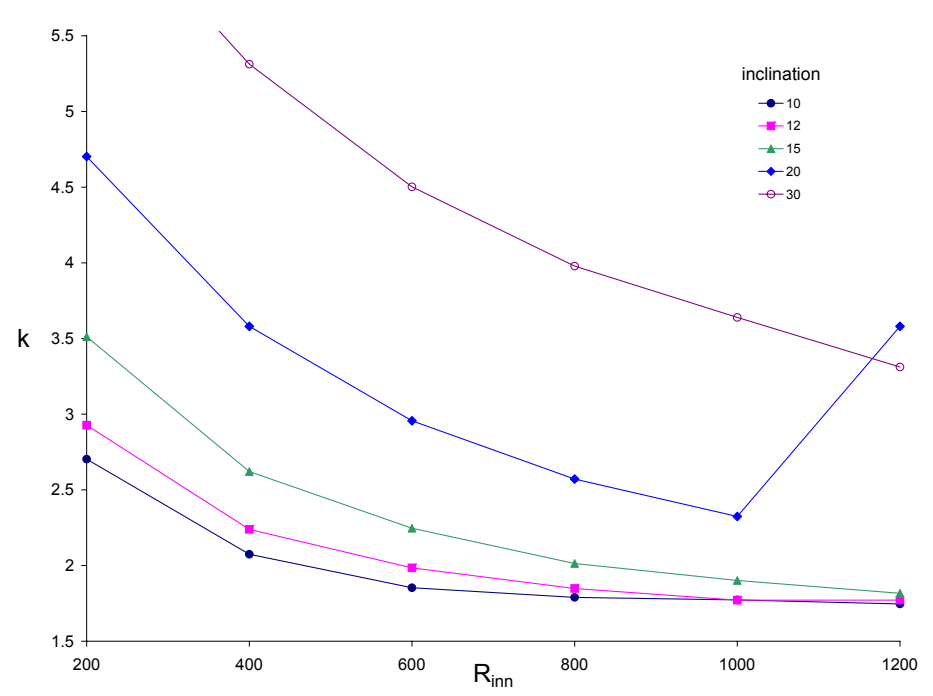

Figure 1. Response of the inner radius of the fixed ring in accretion disk $\left(R_{\text {inn }}-R_{\text {out }}=800 R_{G}\right)$, for different inclinations, to the measured parameter $k=W_{1 / 10} / W_{1 / 2}$ of simulated spectra.

Using this technique we found better accuracy than by fitting of the line shapes. Large number of parameters in the fit causes that we could get only rough estimation of the model parameters (as already shown in Popović et al. 2004, Bon et al. 2006).

We note that it is really hard to estimate the contribution of the disk emission in the one-peaked line profiles. With this approach we showed that one should take into account disk emission, and also we showed domains where we could expect this influence

As a future work we plane to simulate even denser greed of parameters, for finer determination of parameter domains. We also tend to apply this model on a larger number of AGN spectra.

\section{Acknowledgements}

The work was supported by the Ministry of Science, Technologies and Development of Serbia through the project 146002 .

\section{References}

Bon, E., Popović, L. Č., Ilić, D. \& Mediavilla, E. G. 2006, New Ast. Rev., 50, 716

Chen, K. \& Halpern, J. P. 1989, ApJ, 344, 115

Dumont, A. M. \& Collin-Souffrin, S. 1990, A\&AS 83, 71

Ilić, D., Popović, L. Č., Bon, E., Mediavilla, E. G. \& Chavushyan, V. H. 2006, MNRAS, 371, 1610

Kollatschny, W. \& Bischoff, K. 2002, A\&A, 386, L19

Kollatschny, W. 2003, A\&A, 407, 461

Eracleous, M. \& Halpern, J. P. 2003, ApJ, 599, 886

Murray, N. \& Chiang, J. 1997, ApJ, 474, 91

Popović, L. Č., Stanić, N., Kubičela, A. \& Bon, E. 2001, A\&A, 367, 780

Popović, L. Č., Mediavilla, E.G., Kubičela, A. \& Jovanović, P. 2002, A\&A, 390, 473

Popović, L. Č., Mediavilla, E. G., Bon, E., Stanić, N. \& Kubičela, A. 2003, ApJ, 599, 185

Popović, L. Č., Mediavilla, E.G., Bon, E. \& Ilić, D. 2004, ApJ, 423, 909 